protocols and resumption of vaccination meant that six years after the vaccine was introduced, polio was almost eradicated in the United States.

Much of the scientific establishment closed ranks against Salk. He was given the prestigious Lasker Award for clinical medical research in 1956, but Swedish virologist Sven Gard dealt his Nobel nomination a fatal blow by sneering that the vaccine was a technical advance, not a discovery. Nor was Salk elected to the US National Academy of Sciences. Virologist Albert Sabin bombastic, imperious and galled by Salk's success - continued to develop a live, orally delivered poliovirus preparation. By 1961, Sabin's vaccine had performed well in trials and the American Medical Association began to promote it. Salk's vaccine was, for a time, superseded, and his efforts to improve its potency stymied.

Salk moved on, although he remained involved with the polio vaccine. Influenced by chemist C. P. Snow's 1959 book The Two Cultures and the Scientific Revolution, he launched a research institute integrating social responsibility and the humanities with the biological sciences. The Salk Institute recruited some of the great biologists of the time, including Jacob Bronowski, Francis Crick and Jacques Monod. But Salk was unable to translate his lofty ideals into practical management. His research from the 1960s onwards, on immune responses in cancer, multiple sclerosis and, later, HIV/AIDS, met with ambivalence. He was increasingly derided by the very scientists whom he had recruited.

In many ways, Salk was ahead of his time, notably in public engagement and in his multidisciplinary agenda. A polio vaccine would have emerged without him, but it was his vision and willpower that produced the first, and a descendant of it is still the basis of many public-health programmes. Yet universal polio eradication remains a dream: cases continue to appear in Pakistan, Afghanistan and Nigeria, and have resurged in recent years in Syria.

Tilli Tansey is professor of the history of modern medical sciences at Queen Mary, University of London.

e-mail:t.tansey@qmul.ac.uk

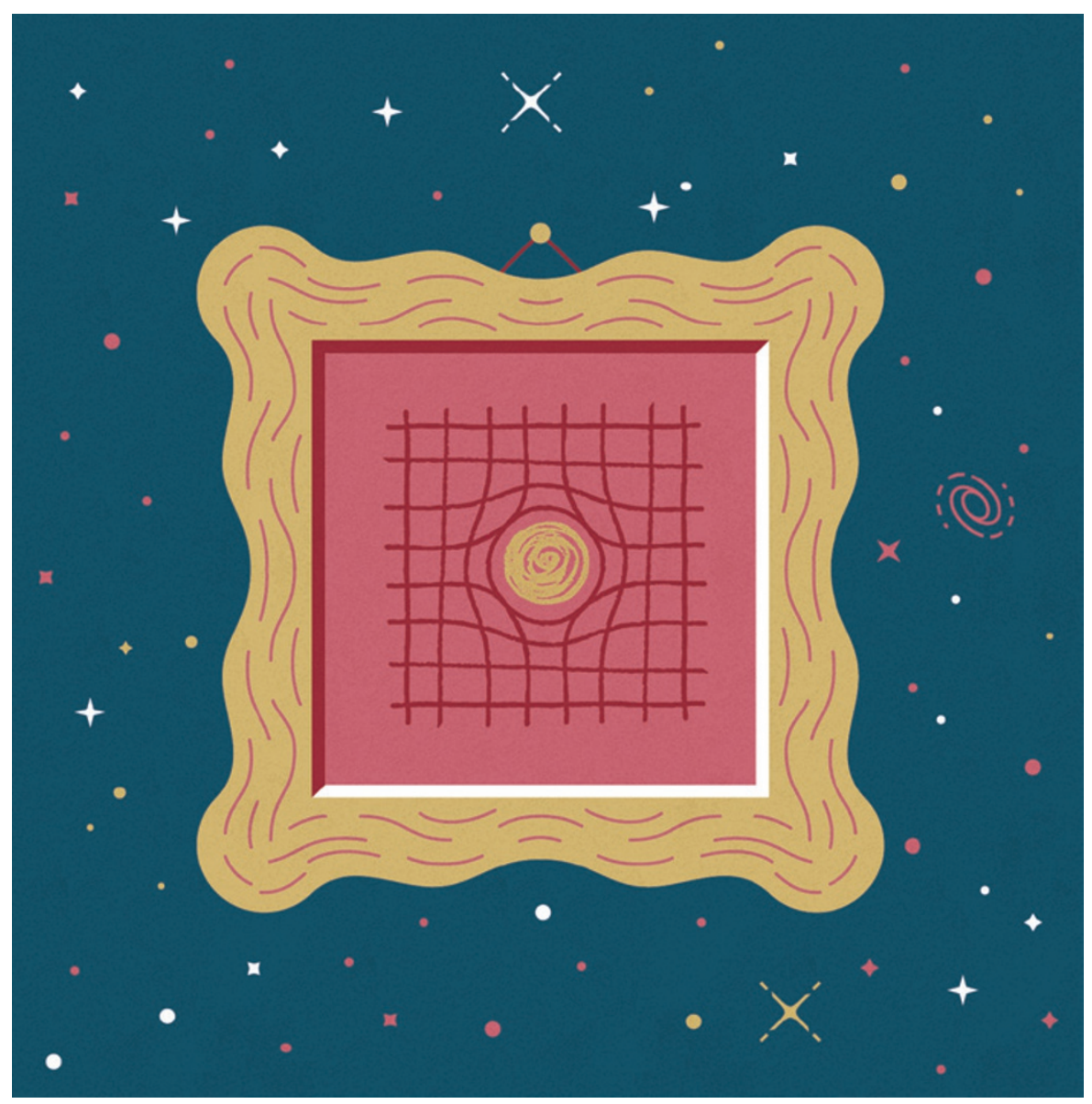

PHYSICS

\title{
One hundred years of general relativity
}

\section{Pedro Ferreira looks back at how Einstein himself and a panoply of other physicists have framed the theory.}

$\mathrm{U}$ ntil very recently, relativists were few and often self-taught. General relativity still had the stigma of being esoteric, pointless and, well, hard. In some places you could find specialized graduate courses, but on the whole, if you were at all interested in expanding universes and black holes, you were left to your own devices. That is what happened to me.

I studied engineering and did not enjoy it very much. But during the course on electromagnetism, I discovered Albert Einstein's world of special relativity.

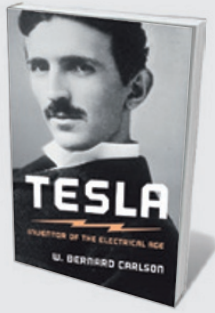

Tesla: Inventor of the Electrical Age

W. Bernard Carlson PRINCETON UNIV. PRESS 2015 Over-hyped eccentric or electricity wizard? Bernard Carlson's account of Nikola Tesla's life at the turn of the twentieth century recalls the inventor's great creations, such as the alternating-current motor, as well as the unfulfilled promise of wireless power. (See Patrick McCray's review: Nature 497, 562-563; 2013.)

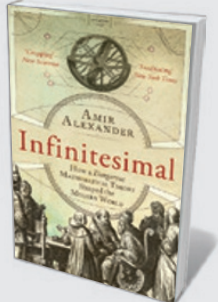

Infinitesimal: How a Dangerous Mathematical Theory Shaped the Modern World Amir Alexander ONEWORLD 2015

Through religious and revolutionary figures of the seventeenth century, Amir Alexander tells the history of the struggle for mathematics' place in society. The 'heretical' concept of infinitesimals, the indivisible points of a line, takes centre stage. 
The mathematics was seductive, the paradoxes were mind-blowing, and it set me up to try to learn his general theory of relativity. That theory explains how the gravitational force is nothing more than space-time bending and warping as it responds to the presence of energy and mass. To understand this revolutionary viewpoint, I had to look for the right book, something that could lead me through all the intricacies of Riemannian geometry, which overturns the rules of Euclidean geometry that we learn in school. Yet I also needed to understand the physics: the bending of light and the orbit of Mercury.

One book stood out: Einstein's popular Relativity: The Special and the General Theory. Published in German in 1916, following Einstein's groundbreaking 1915 paper on the general theory of relativity, it was translated into English in 1920 . In 2015, we see the publication of a special anniversary edition, as well as an annotated version of the manuscript of the paper in Hanoch Gutfreund and Jürgen Renn's The Road to Relativity.

Einstein's book Relativity was supposed to be understandable by all, yet to have enough maths to allow the more educated reader to get into the guts of his ideas. It has very few equations, rendering it less explanatory and more illustrative. But there are definitely a lot of words. Einstein set himself the task of explaining the concepts and ideas behind his theory, using situations from everyday life, such as trains moving on platforms or clocks on walls. His prose is tempered with some philosophical considerations, for example a discussion of the 'a priori' assumption that empty space exists.

Dare I say it, I found the prose inelegant. This caught me by surprise. I had read some of Einstein's 1905 papers, including the one introducing special relativity, and had thought them gems. Relativity, by contrast, was not particularly clear and a bit dull. Einstein had declared, in the introduction, that he would repeat himself frequently, "without paying the slightest attention to the elegance of the presentation". In this he might have been following the dictum of physicist Ludwig Boltzmann, who pinned
Relativity: The Special and the General Theory (100th Anniversary edition) ALBERT EINSTEIN

Princeton Univ. Press: 2015

The Road to Relativity: The History and Meaning of Einstein's "The Foundation of General Relativity" Featuring the Original Manuscript of Einstein's Masterpiece HANOCH GUTFREUND AND JÜRGEN RENN

Princeton Univ. Press: 2015

down the concept of entropy and declared that "matters of elegance should be left to the tailor and to the cobbler". Nevertheless, there is something honest about Einstein's attempt at popular writing: he does not gloss over difficulties. His theory was, to some

\section{BIZARRE PREDICTIONS FROM GENERAL RELATIVITY, PREDICTIONS THAT EINSTEIN WAS WARY OF AT FIRST, HAVE STOLEN HIS THUNDER.}

extent, all there in his book. The treatment just did not seem to work, and he knew it. He told a friend, the Swiss-Italian engineer Michele Besso, that it was "quite wooden". In later years, he joked with the Polish physicist Leopold Infeld that the description "generally understandable" on the book's cover should be changed to "generally not understandable".

Having given up on Einstein, I looked around and found much to choose from. As soon as Einstein had put his theory out, others took over and made it their own. Arthur Eddington, the UK astronomer who had measured the bending of light in a 1919 eclipse expedition, wrote a beautifully crafted mathematical treatise on the theory of space-time in 1923. Erwin Schrödinger, one of the fathers of quantum physics, came up with his own, more-conceptual rendition, Space-Time Structure, published in 1950. Fellow quantum pioneer Paul
Dirac's reticent personality comes across in his lecture notes on the theory, numbering just under 70 pages and published as The General Theory of Relativity in 1975. Euphoria and creativity pour out of the 1,200-page behemoth Gravitation (1973) by John Archibald Wheeler and his disciples Charles Misner and Kip Thorne. I was spoiled for choice.

Although I never used Einstein's book, it kept cropping up in my life. I have a penchant for second-hand bookshops and would keep finding translations, each with its own story. The French version was first translated by Jeanne Rouvière, a protégée of mathematician and politician Emile Borel, and subsequently expanded by Einstein's friend Maurice Solovine. The mathematician Tullio Levi-Civita, whose work had been instrumental in sucking Einstein into Riemannian geometry, recommended an engineer, Giuseppe Luigi Calisse, to do the Italian translation. The Russian version was translated by a Jewish logician-philosopher, Gregorius Itelson, who lived in Berlin and was beaten to death in 1926 by an anti-Semitic crowd.

Today, Einstein's book is a historical curio. I don't think anyone still reads

it as he intended. There have been so many attempts at popularizing the theory, from practitioners and journalists, that anyone can find a book to their taste. And we have learnt much in the century since it was published: a popular book on relativity must now talk about the expanding Universe and the Big Bang, black holes and singularities. These bizarre predictions from general relativity, predictions that Einstein was wary of at first, have stolen his thunder.

Yet I can still see some fugitive magic in Relativity, despite its "wooden" tone. It conjures Einstein as the oracle presenting a theory to the world - one of the most revolutionary and profound theories of all time.

Pedro Ferreira is professor of astrophysics at the University of Oxford, UK, and author of The Perfect Theory.

e-mail:p.ferreira1@physics.ox.ac.uk

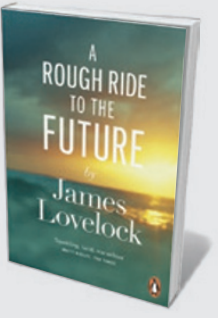

A Rough Ride to the Future

James Lovelock PENGUIN 2015

Independent scientist James Lovelock gazes at

Earth's past, present and future as the selfregulating system Gaia. Focusing on climate, he foresees humanity in 100 million years merged with artificial intelligence to survive a hotter Earth. (See Tim Lenton's review: Nature 508, 41; 2014.)

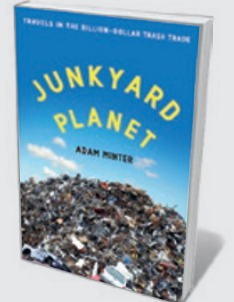

Junkyard Planet: Travels in the Billion-Dollar Trash Trade Adam Minter BLOOMSBURY 2015 One man's trash is another's treasure in Adam Minter's exploration of the US $\$ 500$-billion global recycling trade. US waste tops the charts, and China's electronics-manufacturing industry sifts gold from mountains of e-waste. Emily Banham 